FORMATION Formation emploi

Revue française de sciences sociales

133 | Janvier-Mars 2016

Heurs et malheurs de l'apprentissage en Suisse

\title{
Quand les interactions en formation renforcent les inégalités
}

When interactions during training reinforce inequalities

Wenn Interaktionen in der Ausbildung die Ungleichheiten verstärken

cuando las interacciones en formación refuerzan las desigualdades

Barbara Duc

\section{(2) OpenEdition}

Journals

\section{Édition électronique}

URL : http://journals.openedition.org/formationemploi/4683

DOI : 10.4000/formationemploi.4683

ISSN : 2107-0946

\section{Éditeur}

La Documentation française

Édition imprimée

Date de publication : 20 avril 2016

Pagination : 177-197

ISSN : 0759-6340

\section{Référence électronique}

Barbara Duc, "Quand les interactions en formation renforcent les inégalités », Formation emploi [En ligne], 133 | Janvier-Mars 2016, mis en ligne le 21 avril 2018, consulté le 30 octobre 2020. URL : http:// journals.openedition.org/formationemploi/4683 ; DOI : https://doi.org/10.4000/formationemploi.4683 


\title{
Quand les interactions en formation renforcent les inégalités
}

\author{
BARBARA Duc \\ Docteure en sciences de l'éducation, maître de recherche à l'Institut fédéral des hautes études \\ en formation professionnelle (Lausanne, Suisse)
}

Résumé

\section{Quand les interactions en formation renforcent les inégalités}

Cette contribution s'intéresse aux interactions entre apprenti-e-s et formateurs, durant la période de formation, en Suisse. Il s'agit de mieux comprendre les processus interactionnels en jeu dans la reproduction des inégalités. A cet effet, nous mobilisons une approche qualitative qui s'appuie sur des données audio-vidéo documentant le parcours de formation d'un apprenti automaticien. Une attention particulière est accordée à certaines caractéristiques de cet apprenti qui, au fil de son parcours, deviennent sources de marginalisation et de stigmatisation.

Mots clés : enseignement technique-professionnel, apprentissage, orientation scolaireprofessionnelle, inégalité sociale, socialisation professionnelle, Suisse

Abstract

When interactions during training reinforce inequalities

This contribution is concerned with the interactions between apprentices and trainers during the period of training, in Switzerland. It consists in better understanding interactional processes participating to the reproduction of inequalities. In order to do this, we refer to a qualitative research, which leans on audio-video data documenting the training path of an automation specialist apprentice. A particular attention is paid to some characteristics of this apprentice which become source of marginalization and of stigmatization in the course of his training.

Keywords : technical \& vocational education, apprenticeship, school guidance \& vocational guidance, social inequality, professional socialisation, Switzerland Journal of Economic Literature: I 21, J 15

Traduction : Auteure. 
L'enjeu de cette contribution est de questionner la reproduction des inégalités en formation professionnelle initiale telle qu'elle se joue au cours des situations de formation et en particulier des interactions verbales et non verbales dans lesquelles les apprenti-e-s s'engagent avec les enseignant-e-s, formateurs et formatrices, collègues, autres apprenti-e-s. La présente étude met en évidence les processus interactionnels en jeu dans cette reproduction.

Le système éducatif suisse est considéré comme l'un des systèmes européens les plus sélectifs (Meyer, 2011), et en particulier à deux de ses moments clés : le passage de l'école primaire au secondaire I et le passage du secondaire I au secondaire II. Ces passages sont marqués par des sélections à l'entrée des différentes filières qui composent les degrés secondaires.

Ces sélections ne vont pas sans produire des inégalités puisque le sexe, l'origine sociale et le statut migratoire jouent un rôle prépondérant dans les différentes décisions d'orientation qui ponctuent les parcours des individus (Hupka, 2003 ; Meyer, 2011). Les effets cumulatifs de ces sélections peuvent être illustrés par le cas des jeunes migrant-e-s : indépendamment de leurs performances scolaires, ces jeunes sont souvent plus mal noté-e-s durant l'école primaire déjà, au niveau des différentes évaluations sommatives qui ponctuent le programme de formation. Puis, elles et ils sont fréquemment orienté-e-s vers des filières à exigences élémentaires au moment du passage au secondaire I, ce qui réduit l'éventail des formations post-obligatoires possibles (Kronig, 2007 ; Meyer, Ibid.). Dans ce cas, des caractéristiques sans rapport avec leurs performances, comme l'origine sociale et le statut migratoire, jouent un rôle prépondérant dans la décision de sélection (Kronig, 2007).

Il en va de même au niveau de l'accès au dispositif dual de formation professionnelle initiale, voie la plus fréquemment choisie à la sortie de l'école obligatoire en Suisse (OFFT, 2013). Outre le poids de la filière suivie lors du secondaire I, une grande importance est généralement accordée aux compétences scolaires, en particulier en lecture et en mathématiques (Hupka, 2003). L'origine sociale et le statut migratoire constituent à nouveau des facteurs d'inégalités à l'entrée du dispositif. Les jeunes de classes moins favorisées se retrouvent ainsi deux fois plus nombreux dans les solutions transitoires, durant une, voire deux années, après la fin de l'école obligatoire (Meyer, Ibid.). Il s'agit de programmes de formation (année scolaire supplémentaire, préapprentissage, etc.) destinés à des jeunes qui ne sont pas inscrit-e-s dans une formation secondaire II à la fin de l'école obligatoire. Ces programmes ne sont pas sanctionnés par un diplôme, mais visent à augmenter les chances d'accès à une formation post-obligatoire qualifiante. Par ailleurs, les jeunes migrant-e-s d'origine sociale modeste qui suivent des filières à exigences élémentaires au secondaire I souffrent des effets des stéréotypes dont elles et ils sont la cible et qui entravent leur entrée en apprentissage (Fibbi, Kaya et Piguet, 2003).

A cet égard, les recherches menées s'inscrivent souvent dans le cadre de travaux quantitatifs, basés sur le suivi de cohortes. Elles se focalisent principalement sur les moments de transition que constituent les passages de l'école primaire au secondaire I et de l'école obligatoire à une formation post-obligatoire. En outre, elles mettent en évidence les facteurs d'inégalités dans ce contexte, principalement le sexe, l'origine sociale et le statut migratoire. Le niveau 
scolaire et la filière suivie au secondaire I jouent un rôle important dans ces sélections, mais sont aussi vus comme marqués par ces mêmes facteurs d'inégalités.

Jusqu’à présent, peu de travaux, de surcroît dans une approche qualitative, se sont intéressés à ce qui se passe durant la formation (Imdorf, Granato, Moreau et Waardenburg, 2010), et en particulier au sein du système dual de formation professionnelle.

Nous nous inscrivons dans cette problématique en nous appuyant sur une recherche qualitative (Duc, 2012) menée dans une perspective micro-interactionnelle (Filliettaz, de SaintGeorges et Duc, 2008). Celle-ci accorde une place centrale aux interactions verbales et non verbales, tant sur le plan théorique que méthodologique. Les interactions sont considérées comme le lieu d'accomplissement de différents processus, de reproduction des inégalités dans ce cas, et un moyen de les appréhender à travers leur analyse.

Cette approche permettra de mettre en évidence de façon inédite ${ }^{1}$ quelques mécanismes interactionnels en jeu dans les situations de formation qui semblent participer, d'une part, à la mise à l'écart d'apprenti-e-s présentant certaines caractéristiques, d'autre part, contribuer potentiellement à la reproduction des inégalités. En effet, comment les inégalités liées à l'origine sociale, au statut migratoire et au niveau scolaire en particulier, sont-elles rejouées au cœur des situations de formation et des relations entre les apprenti-e-s et les personnes qu'elles et ils côtoient, en école ou en entreprise ? Quels sont les processus interactionnels en jeu ? Comment certaines caractéristiques des apprenti-e-s sont-elles convoquées pour les qualifier ou les disqualifier et devenir ainsi sources d'inégalités?

La première partie de la contribution est consacrée à la présentation des éléments théoriques et méthodologiques d'une telle approche. $\mathrm{Y}$ est proposée une perspective située et interactionnelle de la socialisation professionnelle, selon laquelle la socialisation s'opère à travers la participation aux pratiques d'une communauté et en particulier aux interactions verbales et non verbales qui y prennent place. Cette perspective a des conséquences sur le plan méthodologique (observation de situations de formation, enregistrement de données audio-vidéo, transcription, analyse des interactions verbales et non verbales) qui seront également développées. Les parties suivantes présentent une étude de cas basée sur l'analyse de données audio-vidéo documentant le parcours de formation d'un apprenti automaticien, et sa discussion. Une attention particulière est accordée à certaines caractéristiques de cet apprenti (niveau scolaire et nationalité), afin de montrer comment elles deviennent sources de marginalisation et de stigmatisation au fil de son parcours. Ce cas est emblématique eu égard aux facteurs d'inégalités en jeu dans le système suisse de formation professionnelle et à la visibilité des mécanismes interactionnels dont nous postulons qu'ils participent à leur reproduction. Enfin, nous revenons en conclusion sur la perspective adoptée et sur la façon dont elle révèle certains des processus fins en jeu dans la perpétuation des inégalités au sein de la formation professionnelle.

1. Il s'agit d'une relecture d'une étude de cas analysée dans le cadre d'un travail de doctorat (Duc, 2012). 


\section{Encadré 1 : Le système éducatif suisse}

Le système suisse d'enseignement est organisé en quatre degrés : le niveau primaire, d'une durée de huit ans; le secondaire I, organisé autour de différents types d'enseignement de degré d'exigence distinct, d'élémentaire à étendue, d'une durée de trois ans ; le secondaire II, constitué de trois filières de formation post-obligatoire, générale, gymnasiale et professionnelle, de durée variable ; et le tertiaire qui regroupe les universités et les hautes écoles spécialisées.

La formation secondaire I fait partie de l'école obligatoire et est sous la responsabilité des cantons. Les élèves y entrent à 12-13 ans et sont réparti-e-s dans des cours à niveaux pour toutes ou une partie seulement des matières enseignées, selon leurs résultats de fin d'école primaire. Les données de notre recherche ont été collectées dans le canton de Genève ; le secondaire I y est organisé en trois regroupements $(R 1, R 2, R 3)$ qui représentent respectivement les filières à exigences élémentaires, moyennes et étendues.

La formation secondaire II fait partie de la formation post-obligatoire et est unifiée sur le plan fédéral. Elle est composée de trois filières que l'on peut intégrer à partir de 15-16 ans. Les filières d'enseignement général et gymnasial préparent à l'obtention d'une maturité spécialisée ou gymnasiale et à l'entrée dans une formation de degré tertiaire. La filière professionnelle prépare à l'obtention d'une attestation fédérale de formation professionnelle (AFP) sur deux ans ou d'un certificat fédéral de capacité (CFC) sur trois à quatre ans. Ces deux diplômes permettent d'entrer directement sur le marché du travail ; le CFC donne aussi accès à une formation professionnelle supérieure de degré tertiaire après l'obtention d'une maturité professionnelle. La filière professionnelle peut être suivie en école professionnelle (école de métiers ou de commerce) à temps complet ou dans le système dual de formation professionnelle initiale caractérisé par l'alternance entre des temps de formation en école et en entreprise.

L'entrée dans ces différents dispositifs dépend en grande partie de la filière suivie au secondaire I et des compétences scolaires (Meyer, 2011).

\section{Le rôle des interactions dans la reproduction des inégalités en formation}

Sur le plan théorique, l'approche proposée est une perspective située et interactionnelle de la socialisation professionnelle. Les deux ingrédients principaux d'une telle perspective - la participation à des communautés de pratique, l'accomplissement des phénomènes de participation et d'identification en interaction - permettent d'aborder la reproduction des inégalités sous l'angle des processus interactionnels qui y participent. 


\subsection{La socialisation professionnelle : participation à des communautés de pratique et identification}

Les systèmes de formation professionnelle, et en particulier les dispositifs en alternance, sont considérés comme des instances de socialisation professionnelle (Dubar, 1996 ; Moreau, 2003). La socialisation professionnelle est envisagée ici dans une perspective située, basée sur la prise en compte des situations sociales dans lesquelles elle se déploie ${ }^{2}$.

Une place centrale est accordée à la théorie des communautés de pratique (Lave et Wenger, 1991), telle qu'elle a été appliquée au processus d'apprentissage par ses auteurs. Dans cette perspective, apprendre est indissociable de la socialisation de nouveaux membres aux pratiques d'une communauté, socialement et culturellement organisée, à travers leur participation à ces activités. Cette participation évolue dans le temps en progressant d'une participation périphérique (tâches mineures et conditions atténuées en termes de responsabilité, rythme, etc.) à une participation pleine.

La participation périphérique légitime désigne cette trajectoire et révèle un aspect central de celle-ci, la légitimité, qui renvoie à l'importance de la reconnaissance des novices en tant que membres de la communauté. Légitimité et reconnaissance révèlent les aspects identitaires des processus d'apprentissage et de socialisation : apprendre implique de devenir un certain type de personne selon les possibilités offertes par la communauté et ne peut être dissocié de la façon dont les participant-e-s deviennent identifiables et sont identifié-e-s dans la communauté (Lave et Wenger, 1991).

L'identification, en tant qu'action sociale, où l'attribution identitaire est extérieure à la personne (Avanza et Laferté, 2005), est centrale dans la perspective adoptée, puisqu'elle est considérée comme un des processus potentiellement en jeu dans la reproduction des inégalités.

Cependant, trois aspects de la théorie des communautés de pratique demandent encore à être discutés.

Premièrement, la connotation positive généralement véhiculée par le terme de communauté (harmonie, intérêts communs) peut être questionnée au regard des réalités du monde du travail. En effet, ce terme ne semble pas prendre en compte les conflits personnels ou d'intérêts, la compétition, les discriminations et autres violences qui peuvent être à l'œuvre au sein des collectifs de travail, que ce soit entre collègues ou personnes de rang hiérarchique différent (Fuller, 2007 ; Jewson, 2007).

2. La notion de «socialisation professionnelle» désigne la socialisation secondaire (Berger et Luckmann, 1996) qui prend place en milieu professionnel et est considérée comme une habituation au monde du travail. Ce processus de socialisation recouvre différentes composantes, à savoir la transmission et l'intégration de savoirs, savoir-faire et savoir-être propres à une activité professionnelle (Dubar, 1996) ; la confrontation à des normes, valeurs, règles, codes propres à un domaine d'activité (Dubar, Ibid.); l'habituation à l'organisation et à la division du travail, à la hiérarchie ainsi qu'aux conditions de travail et d'emploi (Moreau, 2003). 
Deuxièmement, ce modèle théorique peut non seulement servir à expliquer l'apprentissage, illustré par des trajectoires menant à une participation pleine et reconnue, mais aussi son échec. Toute communauté n'est donc pas nécessairement aussi bienveillante que le terme lui-même le laisse présager, à savoir une communauté dans laquelle la formation d'identités positives (positively valued identities) serait possible et attendue pour tous et toutes les participant-e-s (O’Connor, 2003).

Finalement, il est souvent reproché à ce modèle de ne pas prendre suffisamment en compte les relations et les inégalités de pouvoir - associées aux catégories d'âge, sexe, nationalité, classe - en jeu dans une communauté et qui influent sur la socialisation des individus ainsi que sur les phénomènes de participation ou de non-participation (Barton et Tusting, 2005). Ce manque d'attention peut être rapporté à la quasi-absence de prise en compte de l'emboîtement des communautés de pratique dans des contextes sociaux plus larges, et de leur influence sur le fonctionnement des communautés (Contu et Willmott, 2003). Dans la façon dont est appréhendé ici le modèle des communautés de pratique, à savoir sur un plan local, pour désigner les environnements ou les collectifs de travail dans lesquels les apprenti-e-s s'engagent dans le contexte de leur apprentissage, la communauté que forment les employé-e-s d'une entreprise ou les apprenti-e-s d'une classe doit être prise en compte comme emboîtée dans son contexte institutionnel (entreprise, école professionnelle) mais aussi dans le contexte social plus global.

Malgré ces limites, on retiendra néanmoins l'approche située du modèle des communautés de pratique. Les phénomènes de participation et leur dimension identitaire constituent une entrée fructueuse pour mieux comprendre ce qui se passe durant la période de formation et mettre en évidence les processus en jeu dans la reproduction des inégalités.

La prise en compte des aspects langagiers et interactionnels de la participation à des communautés de pratique (Barton et Tusting, 2005) permettra de définir des catégories d'analyse et de saisir plus finement ces processus.

\subsection{Des dynamiques de participation et d'identification à l'œuvre dans l'interaction}

L'appartenance et la participation à une communauté de pratique sont en permanence accomplies, ratifiées, négociées dans les interactions qui s'y déroulent (Rock, 2005). Par conséquent, une attention soutenue est accordée aux interactions, verbales et non verbales, dans lesquelles les apprenti-e-s s'engagent avec les différentes personnes qu'elles ou ils rencontrent au sein des divers environnements de formation et de travail du dispositif dual (enseignant-e-s, formateurs et formatrices, collègues, apprenti-e-s) (voir encadré 2). La perspective adoptée ici se situe ainsi à l'interface entre, d'une part, des approches centrées sur les processus d'apprentissage et de socialisation professionnelle et, d'autre part, des approches centrées sur les phénomènes interactionnels (Filliettaz et al., 2008). 


\section{Encadré 2 : Les données audio-vidéo analysées}

Les données mobilisées ici ont été recueillies dans une démarche ethnographique multi-site (Marcus, 1995) à travers l'observation de situations ordinaires de formation dans les trois lieux du dispositif dual (école professionnelle, entreprise, cours interentreprises (1)) dans le canton de Genève.

Des apprentis (2) de trois filières de formation techniques (mécanique automobile, électricité, automation) ont été suivis à différents moments de leur parcours de formation (entre leur première et leur dernière année d'apprentissage) dans une perspective longitudinale.

Une attention particulière a été accordée aux situations d'interaction entre les apprentis et les différents acteurs du dispositif dual (enseignants en école professionnelle, formateurs en cours interentreprise et en entreprise, collègues, autres apprentis). Afin de conserver des traces de ces observations et d'accéder de manière détaillée aux interactions dans leurs dimensions verbales et non verbales, des enregistrements audio-vidéo ont été réalisés (environ 130 heures) en complément des notes de terrain. Ces enregistrements ont été réalisés en prenant en compte les biais potentiels dus à la présence d'une caméra ainsi qu'en cherchant à atténuer le caractère potentiellement intrusif de celle-ci et à neutraliser le plus possible son effet sur les comportements des acteurs observés (respect d'une période de familiarisation mutuelle avant de filmer; non-utilisation des premiers enregistrements).

Pour les besoins de la recherche, un important travail de réduction et de sélection des données a été effectué (Duc, 2012). Au final, les parcours de formation de deux apprentis, Michel et Rodrigo (3), ont paru particulièrement pertinents en raison des aspects non linéaires qu'ils présentent - échec aux examens intermédiaires, passage du dispositif dual à l'école à plein temps, réorientation à la suite du certificat fédéral de capacité (CFC) -, caractéristiques des trajectoires d'apprentissage dans la formation professionnelle initiale duale (Bergman, HupkaBrunner, Keller, Meyer et Stalder, 2011). En ligne avec la dimension dynamique de l'objet d'étude (évolution des modalités de participation, transformation du positionnement des apprentis dans l'interaction), des séries d'épisodes formant des trajectoires ont été retenues, que ce soit sur le temps court d'une activité (ex : la réparation par soudure d'une pièce métallique d'un châssis de voiture) ou sur le temps plus long d'un projet mené par un apprenti au cours d'un stage (ex: la conception et la réalisation d'un tableau électrique). Les trajectoires mettant en jeu des phénomènes d'évolution de la participation des deux apprentis telles que documentées par les données à disposition ont été privilégiées.

Les séquences d'interaction composant les trajectoires ont ensuite été transcrites (Filliettaz et al., 2008) puis analysées dans une perspective interactionnelle, au moyen des outils d'analyse présentés ci-dessous (rôle, face, place).

1 : Le masculin est retenu ici étant donné que les apprentis rencontrés sont tous de sexe masculin, ce qui reflète la présence majoritaire des garçons dans les filières de formation choisies. Cette remarque est valable pour les autres acteurs du dispositif dual rencontrés.

2 : Les cours interentreprises sont des cours ponctuels organisés par les organisations du monde du travail afin de compléter l'enseignement en école et la pratique en entreprise.

3 : Il s'agit de prénoms d'emprunt, comme ce sera le cas pour les autres acteurs cités par la suite. 
A la participation à une communauté de pratique est associée ici la participation appréhendée à partir des travaux de Goffman $(1973$; 1974) et d'autres travaux rattachés au champ de la linguistique du discours et de l'interaction (Goodwin et Goodwin, 2004. Dans cette perspective, une façon de traiter les modalités de participation des apprenti-e-s aux pratiques d'une communauté est de prendre en compte les identités endossées dans les interactions verbales et non verbales dans lesquelles elles et ils s'engagent.

Il s'agit de leurs identités situées (Zimmermann, 1998), qui se rapportent à une situation et définissent leurs modalités de participation aux actions conjointes, dans lesquelles elles et ils sont engagé-e-s. Pour étayer ce point, sont convoquées les notions de rôle (Filliettaz, 2006 ; Goffman, Ibid.), de face (Goffman, 1974) et de place (Flahault, 1978 ; Kerbrat-Orecchioni, 1992).

La notion de rôle se rapporte aux contributions de chacun des interactant-e-s à l'action collective dans laquelle elles et ils sont engagés. Elle recouvre à la fois les rôles sociaux (ex : apprenti-e, formateur ou formatrice) qui pré-existent à l'engagement dans l'interaction et régissent les conditions d'accès à l'activité, et les rôles praxéologiques, actions participatives à l'interaction, que ce soit sur le plan verbal ou non verbal, dans lesquels ils s'actualisent. Ces derniers sont à la fois déterminés de façon externe (rôles sociaux), et mis en œuvre et négociés localement dans les interactions.

La notion de face est «la valeur sociale positive qu'une personne revendique effectivement à travers la ligne d'action que les autres supposent qu'elle a adoptée au cours d'un contact particulier" (Goffman, 1974, p. 9). Elle est au centre d'un travail de figuration, par lequel les participant-e-s à un échange cherchent à véhiculer une image positive d'elles-mêmes et d'eux-mêmes et à la faire reconnaître.

Enfin, la notion de place se réfere au positionnement des participant-e-s à un échange. Ce positionnement ne peut qu'être envisagé en termes de rapport, désignant à la fois la place occupée par un-e participant-e et la place assignée de façon réciproque aux autres participant-e-s (Flahault, 1978). Le rapport de places renvoie aussi à l'axe vertical invisible qui structure la relation interpersonnelle des participant-e-s à un échange (Kerbrat-Orecchioni, 1992), et implique que les participant-e-s puissent être positionné-e-s dans une place haute de dominant ou basse de dominé. A nouveau, les places sont déterminées par le contexte socio-institutionnel (rôle social, âge, compétence, etc.) mais sont aussi l'objet de négociations permanentes en interaction.

Ces trois notions de rôle, face et place partagent ainsi trois caractéristiques centrales : leur caractère dynamique, négocié et réciproque, puisque la prise de rôle et de place ainsi que la gestion de la face peuvent faire l'objet de négociation ou de renégociation au cours de l'interaction et ont un impact dans le positionnement des autres participant-e-s.

Elles sont considérées comme des ressources permettant à la fois aux interactant-e-s de négocier leur position dans l'interaction et à l'analyste de rendre compte de ce positionnement. Elles permettent de traiter le processus d'identification tel qu'il se joue en interaction 
(Bucholtz et Hall, 2005), en mettant aussi l'accent sur la façon dont les apprenti-e-s sont positionné-e-s par les personnes avec qui elles et ils interagissent.

Dans la suite de l'étude, les notions de rôle, face et place seront donc utilisées comme des catégories d'analyse pour rendre compte des modalités de participation et du positionnement des apprenti-e-s dans les interactions verbales et non verbales dans lesquelles elles et ils s'engagent. Une attention particulière sera accordée aux ressources interactionnelles et multimodales (marqueurs linguistiques, prosodie, gestes, postures, etc.) sur lesquelles repose la participation au fil de l'enchaînement des tours de parole d'une interaction (Goodwin et Goodwin, 2004).

Cependant, malgré les négociations au fil du temps et les interactions dont les rôles, face et place d'un individu peuvent faire l'objet, ceux-ci n'en restent pas moins en partie déterminés par le contexte socio-institutionnel dans lequel prennent place les interactions. Cela ne manque pas d'influencer, voire dans certains cas d'entraver la négociation, par les interactant-e-s, de leur positionnement. L'étude de cas proposée permettra de revenir plus précisément sur ces points.

Cela rejoint par ailleurs la façon dont est envisagée ici l'articulation entre ce qui se passe en interactions (comportements individuels et interindividuels) et les structures sociales plus larges. Prenant en compte les contextes de niveaux différents dans lesquels prennent place les interactions - le contexte local, une classe considérée comme une communauté de pratique dans le cas analysé ci-dessous; le contexte institutionnel, l'école professionnelle ; le contexte social global (Mehan et al., 1986) -, ces structures sociales sont considérées comme enchâssées et s'informant mutuellement (Cicourel, 1981 ; Lemke, 2000 ; Mehan et $a l$. ., 1986). En ce sens, les structures de plus hauts niveaux ont un pouvoir configurant mais pas entièrement déterminant sur ce qui se passe au niveau des interactions ; les structures sociales plus larges sont à leur tour reproduites ou transformées par ces pratiques. Le positionnement adopté se veut ainsi quelque peu hybride. Une place centrale est en effet accordée aux interactions, vues comme un lieu de construction de la signification de l'action, sans toutefois faire une interprétation radicale de ce principe (Filliettaz et al., 2008) et tout en mettant en évidence l'influence des contextes sociaux, du local au plus global, sur les interactions ${ }^{3}$.

\subsection{Saisir des dynamiques de participation et d'identification à travers des trajectoires situées}

Un dernier point demande à être présenté afin de clarifier l'appréhension des phénomènes de participation et d'identification. En effet, afin de mieux comprendre les dynamiques de ces phénomènes (Bucholtz et Hall, 2005), il semble important de ne pas se centrer sur

3. Nous nous distançons en ce sens de la posture ethnométhodologique radicale qui tend à considérer les structures sociales comme des productions locales uniquement, produites dans les interactions. 
des séquences uniques d'interaction, approche la plus fréquemment adoptée. La notion de trajectoire située de participation (de Saint-Georges et Filliettaz, 2008) permet d'ouvrir l'empan temporel d'analyse pour prendre en compte l'enchaînement d'événements plutôt que des situations d'échanges isolées (voir encadré 3).

\section{Encadré 3 : Les trajectoires situées de participation}

S'adossant au modèle de trajectoire développé par Strauss (1992) lors de recherches menées dans le milieu hospitalier, les trajectoires situées constituent un outil méthodologique qui permet de tracer un ingrédient de la situation (un objet matériel, un participant, un objet de savoir, etc.) dans son déploiement temporel et sur des empans temporels variables. Elles sont composées d'épisodes dont l'enchaînement constitue la trame de la trajectoire (voir Figure 1). II s'agit d'épisodes à propos desquels existent des traces détaillées, des enregistrements audiovidéo dans ce cas. Ils sont mutuellement configurants : un épisode peut avoir un pouvoir configurant sur des épisodes à venir et être reconfiguré par ces mêmes épisodes. Ces configurations sont opérées par des ressources sémiotiques variées.

Figure 1: Enchaînement des épisodes d'une trajectoire située et rapport de co-configuration

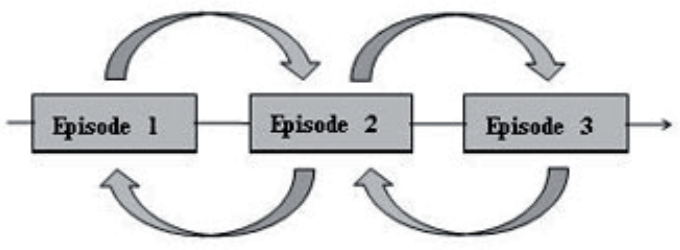

Les trajectoires ne possèdent pas de structure fixe. Elles se déploient dans le temps selon une structuration émergente, en évoluant en fonction des dynamiques sociales et interactionnelles qui les constituent.

Dans le cas présent, les trajectoires situées de participation permettent de tracer et d'analyser les dynamiques de participation et d'identification des apprenti-e-s dans les différents environnements du dispositif dual, sur le temps court de l'activité et sur le temps long du cursus de formation. Cette double attention met en évidence la dimension longitudinale de cette étude. A partir de l'analyse des trajectoires de participation qui s'opèrent à différents moments du parcours des apprenti-e-s, est aussi appréhendée leur trajectoire de participation globale qui se déploie du début à la fin de leur formation

Tout comme l'accent mis sur l'ordre de l'interaction n'empêchait pas la prise en compte de l'influence des structures sociales plus larges sur cet ordre local, ce modèle de trajectoire 
n'exclut pas la prise en compte de l'influence des structures sociales plus larges dans lesquelles elles se déroulent (Lemke, 2000).

Une étude de cas a été retenue ici, basée sur le suivi d'un apprenti au fil de son parcours de formation. Il s'agit de mettre en évidence des dynamiques de participation et d'identification propres à cet apprenti, et d'éclairer comment certaines de ses caractéristiques sont convoquées dans les interactions et influent sur sa participation. Cela permettra de révéler les processus interactionnels en jeu dans la reproduction des inégalités.

\section{Dynamiques de participation et d'identification d'un apprenti automaticien}

L'étude de cas mobilisée ici concerne un apprenti automaticien, Rodrigo, suivi durant son parcours de formation. Elle reprend les analyses des trajectoires situées de participation qui documentent et caractérisent certaines étapes de son parcours. La première partie revient sur les premiers mois de formation de Rodrigo ; la deuxième se penche sur les étapes suivantes de son parcours de formation. Ces éléments d'analyse permettront finalement d'identifier les tendances générales de sa trajectoire de participation globale.

Rodrigo est un apprenti automaticien de première année au moment où commence son suivi. Son parcours scolaire précédant son entrée en formation professionnelle initiale n'est pas linéaire. A la suite de la séparation de ses parents, il alterne entre des périodes passées à Genève, où vit son père qui occupe des emplois peu qualifiés (ouvrier dans une laiterie, agent de sécurité), et des périodes passées au Cap Vert, son pays d'origine, auprès de sa grand-mère maternelle $e^{4}$. Le français n'est donc pas sa langue maternelle. Sa scolarité obligatoire suit ses différents déménagements.

Après avoir terminé sa formation en école obligatoire dans son pays d'origine, il revient à Genève à 17 ans afin de commencer une formation secondaire post-obligatoire. En raison de difficultés relationnelles avec son père, il quitte le logement familial et s'installe dans une résidence d'étudiants peu après le début de sa formation. Découragé par son père d'entreprendre une formation dans la filière d'enseignement général, il opte pour le dispositif dual de formation professionnelle initiale. Il aurait rencontré des difficultés pour accéder à la filière d'enseignement général en raison de son niveau de français et de ses compétences scolaires ; elles lui valent d'ailleurs de participer, avant d'entrer dans le dispositif dual, à un programme de préapprentissage d'une année, comportant des cours de remise à niveau scolaire et des ateliers de pratique professionnelle ainsi que des stages en entreprise. Par ailleurs, il correspond à un profil de jeune (nationalité étrangère, origine sociale modeste, parcours scolaire discontinu, non fréquentation d'une filière du secon-

4. Sa mère est installée aux Etats-Unis. Les informations manquent à son sujet. 
daire I) plus facilement orienté vers la filière professionnelle ( $c f$. introduction). Ainsi, après avoir échoué à l'examen d'entrée en apprentissage d'installateur-électricien, il opte pour la formation d'automaticien, dont il réussit de justesse l'examen d'entrée (tests de mathématiques, français, logique et raisonnement). La formation d'automaticien exige de nombreuses connaissances et compétences dans différents domaines (électricité, électronique, mécanique générale, etc.). Elle est considérée comme une formation à fortes exigences (Stalder, 2005). Rodrigo peine ensuite à trouver une place d'apprentissage. Après avoir contacté une vingtaine d'entreprises, sans succès, il se présente comme stagiaire dans une entreprise qui lui proposera finalement une place d'apprentissage. Rodrigo commence alors son apprentissage ; il est âgé de 18 ans, l'âge moyen d'entrée en formation professionnelle à Genève (Rastoldo, Evrard et Amos, 2007).

Le profil de Rodrigo est représentatif d'une partie des jeunes engagés dans la formation professionnelle duale : scolarité obligatoire et transitions non linéaires (discontinuité, passage par un programme de préapprentissage), nationalité étrangère et français comme langue seconde (Rastoldo et al., 2007).

\subsection{Dynamiques de participation régressives et catégorisation comme un apprenti faible}

La première trajectoire de participation retenue pour les besoins de ce travail concerne les premiers mois de formation de Rodrigo. Il fréquente un centre interentreprises, pour une durée de six mois, en alternance avec l'école professionnelle. Il s'agit d'une période de formation suivie par un groupe d'une dizaine d'apprentis automaticiens de première année. Leur sont dispensés des cours théoriques et pratiques qui portent sur les notions et les gestes de base de la mécanique générale et de l'électricité, en amont de leur première expérience en entreprise.

Selon les données sélectionnées, Rodrigo participe à une activité liée au domaine de l'électricité durant le cinquième mois de formation, soit l'assemblage d'un tableau électrique. Il s'agit d'un exercice que l'apprenti doit réaliser de façon individuelle. Dans ce cas, Rodrigo est accompagné par le formateur à différentes étapes de sa réalisation. Notre analyse se centre sur les interactions qui en découlent, avec le formateur ou les autres apprentis présents dans le même espace de formation.

Nous avons privilégié ici une synthèse de la présentation et l'analyse des trois épisodes qui forment la trajectoire, sous la forme d'une vignette narrative (voir encadré 4). Elle est suivie par une analyse synthétique de la trajectoires.

5. Pour le détail des analyses, voir Duc (2012). 


\section{Encadré 4 : Vignette narrative de la trajectoire de participation : activité d'assemblage (Film n' 216,02 '16-01'01'40 et 217, 00'06-35'16)}

Rodrigo est engagé dans un exercice d'assemblage électrique d'un appareil de commande servant à actionner un moteur.

L'exercice se déroule en plusieurs étapes : mise par écrit de l'énoncé donné oralement par le formateur ("Je veux que lorsque j'appuie sur le bouton start le moteur s'enclenche ainsi qu'un voyant lumineux, etc. ») ; dessin du schéma électrique ; assemblage du circuit électrique. Rodrigo est en retard par rapport au reste du groupe dans la réalisation de cet exercice et a de la difficulté à comprendre la première étape de rédaction. Le formateur intervient auprès de lui et il en découle une interaction dans un espace partagé par les autres apprentis, ce qui permet la participation de certains d'entre eux à l'interaction en cours. Rodrigo progresse difficilement dans la tâche et le formateur intervient à plusieurs reprises auprès de lui, que ce soit pour l'étape de rédaction mais aussi pour celle du dessin du schéma électrique. Ses difficultés sont manifestes (difficultés de compréhension, lacunes théoriques). Le formateur y répond tout d'abord par un guidage accru, puis prend progressivement en charge la tâche en cours en évaluant de façon négative l'état des connaissances de Rodrigo à travers des remarques ironiques (" t'as tout oublié hein, c'est pas toi qui m'as dit que tu devais manger beaucoup pour pas oublier [...] t'as pas beaucoup mangé ces temps-ci hein » (Film no 216, 04'41); "t'es malade, t'as besoin qu'on te prenne la température toi » (Film no 216, 24'01). A cela s'ajoute la participation des autres apprentis en écho aux réactions du formateur, qui rient et raillent Rodrigo à leur tour (" ça, c'est la playstation start, t'appuies sur select, ça s'arrête » (Film no 216, 03'30) ; «faut pas copier Cap Vert » (Film no 216, $\left.55^{\prime} 50\right)$; « il est marrant ce Cap Vert » (Film no 217, 04'50).

Les interactions entre Rodrigo et le formateur, qui composent les épisodes sélectionnés, marquent une rupture par rapport à celles qui se déroulent entre eux durant les premiers mois de formation dans le centre interentreprises. Les situations de formation observées auparavant montrent l'accompagnement soutenu de Rodrigo par le formateur au cours d'activités liées au domaine de la mécanique : adaptation au niveau de compétence de l'apprenti et prise en compte de ses difficultés (oublis, difficultés arithmétiques), familiarisation progressive aux tâches et prise en charge croissante de celles-ci au moyen des ressources mises à disposition par le formateur.

Dans le cas retenu ici, la participation de Rodrigo se caractérise plutôt par une tendance régressive. En effet, le formateur prend progressivement en charge les tâches en cours en tant qu'acteur principal. Rodrigo se voit peu à peu attribuer le rôle de spectateur, et ce malgré ses tentatives de résistance. Elles peuvent être illustrées par la situation d'interaction suivante (Film n ${ }^{\circ} 216,42$ '27-44’53), au cours de laquelle Rodrigo résiste physiquement à la tentative de prise en charge de la tâche en cours par le formateur (recherche d'une information dans un classeur de référence), en cherchant à conserver le classeur que le formateur essaie de lui enlever des mains. Sa tentative de résistance échoue, le formateur met la main sur le classeur et cherche l'information par lui-même. La prise en charge de la tâche par le formateur s'opère ainsi dans la force et met à jour les tensions qui existent entre les deux interactant-e-s. 
Cette rupture est tout aussi manifeste du côté de la reconnaissance de Rodrigo au sein du groupe, et de la façon dont il est identifié, que ce soit par le formateur ou les autres apprentis. Ses difficultés ainsi que son niveau de compétence relativement faible sont très vite évalués de façon négative par le formateur et font l'objet de remarques ironiques (voir encadré 4). Elles sont par conséquent exposées de façon croissante au regard des autres apprentis qui partagent le même espace de formation (établis disposés dans un espace qui s'apparente à une salle de classe). Ces derniers y réagissent le plus souvent en écho aux réactions du formateur, par des rires ou des railleries (voir encadré 4). Ce faisant, les interventions du formateur et des autres apprentis contribuent à catégoriser (Rochex, 2011) progressivement Rodrigo comme un apprenti faible.

Cela ne manque pas d'affecter sa face et sa place au sein du groupe. Il perd ainsi progressivement le contrôle non seulement sur la tâche en cours, mais aussi sur son positionnement dans l'interaction. En effet, Rodrigo cherche à plusieurs reprises à préserver sa face, que ce soit en justifiant ses difficultés ou en contestant les accusations formulées par le formateur. Il cherche aussi à se défendre des moqueries des autres apprentis en niant certaines de leurs affirmations. Cependant, Rodrigo se retrouve de plus en plus isolé face aux autres participants et ne parvient pas à préserver une image positive de lui-même. A ce propos, il est à noter que si le formateur ne participe pas aux interventions des autres apprentis, il ne réagit pas non plus aux menaces qu'elles constituent pour Rodrigo.

Ces phénomènes peuvent être illustrés par deux situations particulièrement frappantes qui prennent place au sein de cette trajectoire. La première (Film no 216, 55'03-01'01'53 et 217, 00’24-07'21) a lieu devant le tableau noir au centre de l'espace partagé par l'ensemble des apprentis. Le formateur entreprend d'expliquer à Rodrigo le fonctionnement et le branchement d'un composant électrique pour les besoins de l'exercice de dessin de schéma en cours qu'il n'arrive pas à effectuer. Après lui avoir demandé de dessiner un circuit électrique au tableau, le formateur se rend compte de l'étendue de ses difficultés. S'efforçant de guider Rodrigo, il perd patience à plusieurs reprises, ce qui se traduit par la prise en charge de la tâche de dessin et par des commentaires dépréciatifs tels que " t'es vraiment un cas hein " (Film no 216, 01'01'38). A nouveau, les autres apprentis présents réagissent en riant et en ratifiant les évaluations négatives du formateur ("faut pas copier Cap Vert» (Film no 216 , 55’50) ; " il est marrant ce Cap Vert " (Film no 217, 04'50). Ces interventions tendent à cristalliser l'image d'apprenti faible attribuée à Rodrigo, sans que ce dernier parvienne à se défendre et à donner une image positive de lui-même.

Cette catégorisation engendre peu à peu une forme de marginalisation au sein de la classe, ce qu'illustre la deuxième situation d'interaction (Film no 217,12 '52-16'21). Elle consiste en une discussion au sein du groupe à propos de la différence entre automaticiens et câbleurs : les premiers sont vus comme des travailleurs qualifiés en charge des tâches de conception alors que les seconds comme des travailleurs sans diplôme qui assemblent des composants électriques sans comprendre ce qu'ils font. Au cours de la discussion où se dessinent deux groupes distincts, dont l'un est davantage valorisé que l'autre, Rodrigo est 
progressivement assimilé aux " câbleurs " alors que les autres apprentis sont assimilés aux "automaticiens ». Un court extrait de cette discussion (Film no 217,14 '13-14’20) montre comment Rodrigo est explicitement identifié à un câbleur ("Cap Vert il fait que câbler ") alors que les autres apprentis s'identifient à des automaticiens ("nos plans»). En plus de l'assimiler de façon dépréciative à un câbleur, Carlos, un autre apprenti, utilise son origine étrangère qui devient un moyen de le désigner (voir autres exemples de ce type ci-dessus), et un autre de ses collègues, Donald, l'identifie à un "blaireau ", un incapable, ce qui renforce son identification à un câbleur. Par ailleurs, cette identification est à la fois menée par les apprentis (ici Carlos et Donald) et par le formateur qui s'appuie sur les interventions des apprentis tout en les ratifiant ("ouais, mais il câble d'après quoi »).

Rodrigo, témoin de l'interaction, n'y participe pas en tant que locuteur et ne réagit pas aux commentaires dépréciatifs émis à son égard. Son silence peut être interprété comme une conséquence même du processus de marginalisation dont il est l'objet. En raison de son statut de formateur en termes de détention du savoir et du pouvoir, les différentes interventions du formateur dans les interactions collectives (commentaires dépréciatifs, ratification des paroles des autres apprentis), jouent un rôle particulier dans l'identification de Rodrigo.

\subsection{Des phénomènes qui se cristallisent au fil du parcours de formation}

Différentes étapes du parcours de formation de Rodrigo ont été documentées à travers l'analyse de trajectoires (période de formation en entreprise en première année, périodes de formation en école en deuxième et troisième suite à son échec aux examens intermédiaires à la fin de la deuxième année et par conséquent à son passage en école à plein temps, stages en entreprise en fin de formation).

Sans entrer dans les détails des analyses, on est frappé par la représentativité et la récurrence des dynamiques de participation régressives telles que relevées ci-dessus. En effet, malgré la variation des communautés dans lesquelles Rodrigo s'engage (école plein temps, entreprises), certains phénomènes sont récurrents : les difficultés qu'il manifeste dans la conduite des activités (oublis, lacunes théoriques et terminologiques, difficultés de compréhension et de verbalisation) et qui sont rapidement sources de perte de patience pour la personne qui l'encadre, ainsi que la prise en charge, par cette dernière, de la tâche en cours.

Cette logique de substitution s'accompagne souvent de railleries à l'égard de Rodrigo, ce qui met en évidence les formes stigmatisantes que prennent les modalités d'encadrement à son égard. Ces réactions concourent ainsi à la régression de la participation de Rodrigo : perte de contrôle sur l'activité et endossement de rôles d'acteur secondaire (exécutant, observateur). Elles contribuent également à son identification et sa catégorisation comme un apprenti faible et peu autonome.

D’autres réponses sont néanmoins apportées aux difficultés de Rodrigo, dans le cas de l'école professionnelle et d'une entreprise dans laquelle il effectue un stage. Il bénéficie d'un accompagnement soutenu, qui s'adapte à son niveau de compétences. Cependant, l'analyse 
de différentes situations d'interaction révèle que la participation de Rodrigo se caractérise par une prise en charge des tâches qui lui sont confiées, tout aussi fragile que dans les situations précédentes. Rodrigo peine à garder la main sur l'activité, que ce soit en interaction avec son enseignant, son responsable de stage, d'autres employés de l'entreprise ou encore un stagiaire moins expérimenté que lui. Sa participation apparaît à nouveau comme peu progressive et irrégulière.

Par ailleurs, sa tendance à perdre la main sur l'activité peut être corrélée avec sa difficulté à garder le contrôle sur la façon dont il se positionne dans l'interaction, l'image positive qu'il cherche à donner de lui-même, et ce malgré les efforts qu'il entreprend pour préserver sa face ou pour garder une place haute dans l'interaction. Cela peut être illustré par la façon dont la justification ou la contestation de ses difficultés ainsi que ses tentatives pour les dissimuler sont le plus souvent mises en échec dans les différentes situations d'interaction analysées.

La redondance de ces phénomènes dessine ainsi les tendances générales de la trajectoire globale de participation et d'identification de Rodrigo.

\section{Le niveau scolaire et l'origine étrangère de l'apprenti comme stigmates}

A partir de ces éléments, revenons sur deux des caractéristiques de Rodrigo qui jouent un rôle prépondérant dans son identification et sa catégorisation au fil de son parcours de formation.

En effet, au cours des différentes situations d'interaction analysées ci-dessus, c'est tout d'abord le niveau scolaire de Rodrigo (son niveau de connaissances et de compétence, ses difficultés, ses lacunes) qui est convoqué de façon appuyée et évalué de façon négative, que ce soit par la personne en charge de son encadrement ou les autres apprentis. Cela influe fortement sur sa participation aux activités en cours et sur son identification au sein de la communauté de pratique. Cela engendre une participation à tendance régressive et une catégorisation comme un apprenti faible. Dans le cas du cours interentreprises en particulier, cette identification se cristallise au fil des interactions pour aboutir à une forme de marginalisation de l'apprenti par rapport au reste du groupe.

La centralité du niveau scolaire dans la participation et l'identification de cet apprenti peut être mise en évidence, de façon contrastive, à travers les données concernant un autre apprenti du même groupe, Donald, de nationalité suisse et de langue maternelle française et ayant connu un parcours scolaire linéaire. En effet, sans entrer dans les détails des analyses menées à son sujet (Losa, Duc et Filliettaz, 2014) ${ }^{6}$, on observe combien son niveau

6. Dans Losa, Duc et Filliettaz (2014), les données relatives à Donald et à Rodrigo sont analysées de façon 
scolaire élevé (manifeste dans la façon dont il effectue les exercices en cours, les réponses qu'il donne au formateur, les initiatives quili prend, etc.), évalué de façon positive par le formateur au cours de nombreuses séquences d'interaction, lui permet de gagner en visibilité et en centralité, et d'être identifié, à l'inverse de Rodrigo, comme un participant légitime. Par ailleurs, il parvient à négocier son positionnement au sein du groupe d'apprentis, ce qui parait hors de portée de Rodrigo. Cela peut être illustré par les différents moments d'explication collective au cours desquels le groupe est rassemblé et où Donald occupe une position centrale à travers ses réponses et ses initiatives, ratifiées à la fois par le formateur et les autres apprentis. A ce sujet, il est intéressant de mettre en parallèle la façon dont les interventions du formateur participent, dans le cas de Donald, à la construction d'une identité positive (valorisée positivement) de l'apprenti, en comparaison avec ce qui se passe au niveau de l'identification de Rodrigo (voir ci-dessus).

Le rôle que joue aussi l'origine étrangère de Rodrigo dans son identification demande à être discuté ici. En effet, comme dans le cours interentreprise, son origine étrangère est fréquemment évoquée dans les interactions. Le plus souvent utilisée pour le désigner, elle s'articule et participe à l'image d'apprenti faible attribuée à Rodrigo, voire à sa marginalisation. Ce qui est frappant ici, c'est la construction progressive d'un allant de soi, associant faiblesse du niveau scolaire et origine étrangère. Il en résulte une place marginalisée par rapport au reste du groupe. Cette construction est non seulement à rechercher du côté des interactions analysées mais aussi du côté du contexte social plus large dans lequel s'inscrit la communauté de pratique lieu de ces interactions. En effet, la construction de cet allant de soi semble être informée par ce qui se passe en dehors de la communauté de pratique et qui influence son fonctionnement, à savoir les relations et inégalités de pouvoir liées aux catégories de nationalité et de statut migratoire (Hupka, 2003 ; Meyer, 2011). De ce fait, il serait plus judicieux de parler de réactualisation, voire de reproduction, de cet allant de soi au sein des interactions auxquelles participe Rodrigo.

La redondance des phénomènes évoqués et la sédimentation de l'image peu valorisée de Rodrigo comme un apprenti faible incitent à penser son niveau scolaire ainsi que sa nationalité comme des stigmates à partir desquels il est désigné et qualifié négativement (Goffman, 1975). Comme le révèle l'étude de cas, ces stigmates tendent à entraver la participation de Rodrigo au travail de figuration et plus globalement son positionnement dans l'interaction en raison du discrédit qu'ils jettent sur ce dernier (Goffman, Ibid.). Les processus d'identification et de catégorisation, tels qu'ils se déploient en interaction, y jouent un rôle central. C'est en effet à travers ces processus que certaines caractéristiques de l'apprenti, par ailleurs reconnues comme des facteurs d'inégalités, sont convoquées dans l'interaction pour devenir sources de marginalisation et de stigmatisation. Ils participent en ce sens à la reproduction des inégalités en situation de formation.

contrastive, ce qui permet d'ouvrir la réflexion sur ce que valorise ou ce que ne valorise pas la formation professionnelle, en termes de profils d'apprentis, de types de participation ou de positionnement. 


\section{Conclusion}

Il convient de revenir ici sur la perspective adoptée dans cette contribution et les processus en jeu dans la socialisation professionnelle des apprenti-e-s et dans la reproduction des inégalités au sein du système dual de formation professionnelle suisse.

Premièrement, la démarche par étude de cas proposée permet de mieux saisir ce qui se passe durant les périodes de formation dans les institutions en charge de la formation des apprenti-e-s. Létude de cas, choisie pour son caractère emblématique, permet ainsi de mieux comprendre comment certaines caractéristiques d'un apprenti peuvent devenir sources de marginalisation et de stigmatisation, et participer à la reproduction des inégalités dans le système de formation professionnelle suisse.

Deuxièmement, la perspective micro-interactionnelle permet d'éclairer les mécanismes interactionnels qui sous-tendent la construction des dynamiques de participation et d'identification expérimentées par les apprenti-e-s au sein du système dual et qui contribuent à leur socialisation. Participer à une communauté de pratique et se socialiser à ses pratiques apparait ainsi comme un travail collectif qui se joue en interaction : participer, devenir un participant légitime, ou non, c'est aussi participer à des interactions, négocier sa position et l'image que l'on veut donner de soi-même. L'étude de cas proposée analyse la situation d'un apprenti dont les difficultés à se positionner dans l'interaction et à agir sur sa participation sont manifestes. Ces difficultés peuvent être vues comme les conséquences des stigmates dont il est porteur, son niveau scolaire et son origine étrangère, qui sont fortement convoqués dans les situations analysées.

Ces éléments soulignent aussi les niveaux inégaux de ressources et d'autorité que les individus possèdent pour négocier leur position dans l'interaction et pour positionner l'autre. Cela renvoie à la question des communautés de pratique qui doivent être considérées non pas comme des entités favorables à la socialisation et à la construction d'identités valorisées pour tous leurs membres mais comme des communautés emboîtées dans des contextes sociaux plus larges et traversées par des relations et des inégalités de pouvoir, associées à des catégories, comme l'âge, le sexe, la nationalité et la classe sociale.

Ces dimensions amènent par conséquent à penser la participation à une communauté de pratique comme la socialisation du novice - dans ce cas l'apprenti-e - non seulement à l'ordre social de la communauté mais aussi aux contextes sociaux plus larges dans lesquels elle est enchâssée et qui influent sur son fonctionnement. Il s'agit ainsi d'une acculturation à l'ordre social en place et à la position de l'apprenti-e au sein de celui-ci, dans le cas de Rodrigo, une position de dominé, en regard de son niveau scolaire et de son statut d'immigré.

Enfin, le cas traité permet de mettre en évidence le renforcement des inégalités en interaction, objet de l'article. Afin de montrer comment ce qui se passe en interaction peut aussi contribuer non pas à une reproduction mais à une réduction des inégalités, il conviendrait de développer cette analyse en convoquant d'autres cas mettant en scène des apprentis au 
profil similaire ou distinct. Cela permettrait de poursuivre les réflexions entamées ici sur le rapport entre les interactions et les structures sociales dans lesquelles elles s'opèrent, sur le rôle des interactant-e-s dans le renforcement ou l'affaiblissement des inégalités - en partie lié à la façon dont elles et ils convoquent ou non certaines caractéristiques, voire stigmates, des personnes avec lesquelles elles et ils interagissent - et sur leur propre positionnement dans l'interaction.

\section{Bibliographie}

Avanza M. et Laferté G. (2005), «Dépasser la «construction des identités» ? Identification, image sociale, appartenance ", Genèse, n 61, pp. 134-152.

Barton D. et Tusting K. (dir.) (2005), Beyond communities of practice. Language, power and social context, Cambridge, Cambridge University Press.

Berger P. et Luckmann T. (1996), La construction sociale de la réalité, Paris, Armand Colin.

Bergman M.M., Hupka-Brunner, S., Keller, A., Meyer, T. et Stalder, B.E. (dir.) (2011), Youth transitions in Switzerland. Results from the TREE Panel Study, Berne, Seismo.

Bucholtz M. et Hall K. (2005), "Identity and interaction : a sociocultural linguistic approach”, Discourse Studies, vol. 7, n’ 4-5, pp. 585-614.

Cicourel A. (1981), "Notes on the integration of micro- and macro-levels of analysis", in Knorr-Cetina K. et Cicoure A. (dir.), Advances in Social Theory and Methodology-Toward an Integration of Micro- and Macro-Sociologies, pp. 51-80, London, Routledge and Kegan Paul.

Contu A. et Willmott H. (2003), "Re-embedding situatedness : the importance of power relations in learning theory”, Organization Science, vol 14, n 3, pp. 283-296.

de Saint-Georges I. et Filliettaz L. (2008), "Situated trajectories of learning in vocational training interactions", European Journal of Psychology of Education, vol. XXIII, $\mathrm{n}^{\circ} 2$, pp. 213-233.

Dubar C. (1996), La socialisation : Construction des identités sociales et professionnelles, Paris, Armand Colin.

Duc B. (2012), La transition de l'école au monde du travail : une analyse interactionnelle et longitudinale des phénomènes de participation et de construction identitaire en formation professionnelle initiale, Thèse de doctorat en sciences de l'éducation, université de Genève, Genève.

Fibbi R., Kaya B. et Piguet E. (2003), Nomen est omen : Quand s'appeler Pierre, Afrim ou Mehmet fait la différence, Direction du programme PNR 43 en collaboration avec le Forum Formation et emploi et le Centre suisse de coordination pour la recherche 
en éducation (CSRE), Bern/Aarau, consulté le 5 août 2014. URL : http://www.snf.ch/ SiteCollectionDocuments/nfp/nfp43_piguet_synthesis3.pdf

Filliettaz L. (2006), « Asymétrie et prises de rôles. Le cas des réclamations dans les interactions de service ", in Laforest $M$. et Vincent D. (dir.), Les interactions asymétriques, pp. 89-112, Québec, Editions Nota bene.

Filliettaz L., de Saint-Georges I. et Duc B. (dir.) (2008), “"Vos mains sont intelligentes !” Interactions en formation professionnelle initiale ", Genève, Cahiers de la section des sciences de l'éducation.

Flahault F. (1978), La parole intermédiaire, Paris, Editions du Seuil.

Fuller A. (2007), "Critiquing theories of learning and communities of practice", in Hughes J., Jewson N. et Unwin L. (dir.), Communities of practice. Critical perspectives, New York, Routledge, pp. 17-29.

Goffman E. (1973), La mise en scène de la vie quotidienne. La présentation de soi, Paris, Les Editions de Minuit.

Goffman E. (1974), Les rites d'interaction, Paris, Les Editions de Minuit.

Goffman E. (1975), Stigmates. Les usages sociaux des handicaps, Paris, Les Editions de Minuit.

Goodwin C. et Goodwin M. H. (2004), "Participation”, in Duranti A. (dir.), A companion to linguistic anthropology, Malden, Blackwell, pp. 222-244.

Hupka S. (2003), « Situations et parcours de formation », in Office fédéral de la statistique, Parcours vers les formations postobligatoires. Les deux premières années après l'école obligatoire. Résultats intermédiaires de l'étude longitudinale TREE, Neuchâtel, OFS/TREE, pp. 33-57.

Imdorf C., Granato M., Moreau G. et Waardenburg G. (2010), "Vocational Education and Training in Switzerland, France, Germany - Perspectives for Sociological Research", Swiss Journal of Sociology, vol. 36, $\mathrm{n}^{\circ}$ 1, pp. 5-10.

Jewson N. (2007), "Rethinking the concept of community within communities of practice”, in Hughes J., Jewson N. et Unwin L. (dir.), Communities of practice. Critical perspectives, New York, Routledge, pp. 68-82.

Kerbrat-Orecchioni C. (1992), Les interactions verbales, vol. 2, Paris, Colin.

Kronig W. (2007), Die systematische Zuffälligkeit des Bildungserfolgs. Theoretische Erklärungen und empirische Untersuchungen zur Lernentwicklung und Leistungsbewertung in unterschiedlichen Schulklassen, Bern, Haupt.

Lave J. et Wenger E. (1991), Situated learning: Legitimate Peripheral Participation, Cambridge, Cambridge University Press.

Lemke J. (2000), " Across the scales of time: artifacts, activities, and meanings in ecosocial systems ", Mind, Culture and Activity, vol. 7, n 4, pp. 273-290. 
Losa S., Duc B. et Filliettaz L. (2014), "Success, Well-Being and Social Recognition : An Interactional Perspective on Vocational Training Practices", in Bergman M. M., Keller A., Samuel R. et Semmer N. K. (dir.), Success and Well-Being in Education and Employment, Dordrecht, Springer, pp. 69-98.

Marcus G. E. (1995), "Ethnography in/of the world system : the emergence of multi-sited ethnography", Annual Review of Anthropology, n² 24, pp. 95-117.

Mehan H., Hertweck A. et Meihls J.L. (1986), Handicapping the Handicapped. Decision making in students' educational careers, Stanford, California, Stanford Univesity Press.

Meyer T. (2011), "On ne prête qu'aux riches : l'inégalité des chances devant le système de formation en Suisse ", in Bergman M.M., Hupka-Brunner S., Keller A., Meyer T., Stalder B.E. (dir.), Youth transitions in Switzerland. Results from the TREE Panel Study, Berne, Seismo, pp. 40-65.

Moreau G. (2003), Le monde apprenti, Paris, La Dispute.

O’Connor K. (2003), "Communicative practice, cultural production, and situated learning. Constructing and contesting identities of expertise in a heterogeneous learning context", in Wortham S. et Rymes B. (dir.), Linguistic anthropology of education, Westport, Praeger, pp. 61-91.

OFFT (2013), La formation professionnelle en Suisse en 2013. Faits et données chiffrées, Berne, OFFT.

Rastoldo F., Evrard A. et Amos J. (2007), Les jeunes en formation professionnelle. Rapport I : Entrée en formation professionnelle et parcours de formation des diplômés, Genève, SRED.

Rochex J.-Y. (2011), «Au cœur de la classe, contrats didactiques différentiels et production d'inégalités ", in Rochex J.-Y. et Crinon J. (dir.), La construction des inégalités scolaires, Rennes, PUR, pp. 91-110.

Rock F. (2005), “"I've picked some up from a colleague” : language, sharing and communities of practice in an institutional setting", in Barton D. et Tusting K. (dir.), Beyond communities of practice. Language, power and social context, Cambridge, Cambridge University Press, pp. 77-104.

Stalder B. E. (2005), Das intellektuelle Anforderungsniveau von 105 Beruflehren. Internes Arbeitspapier, TREE, Bern.

Strauss A. L. (1992), "Maladie et trajectoires », in Strauss A.L. (dir.), La trame de la négociation : sociologie qualitative et interactionnisme, Paris, L'Harmattan, pp. 143-189.

Zimmermann D. H. (1998), "Identity, context and interaction", in Antaki C. et Widdicombe S. (dir.), Identities in talk, London, Sage Publications, pp. 87-106. 